\title{
Gibberellin homeostasis and plant height control by EUI and a role for gibberellin in root gravity responses in rice
}

\author{
Yingying Zhang ${ }^{1}$, Yongyou Zhu', Yu Peng ${ }^{1}$, Dawei Yan ${ }^{1}$, Qun $\mathrm{Li}^{1}$, Jianjun Wang ${ }^{2}$, Linyou Wang ${ }^{2}$, Zuhua $\mathrm{He}^{1}$
}

${ }^{l}$ National Key Laboratory of Plant Molecular Genetics, Institute of Plant Physiology and Ecology, Shanghai Institutes for Biological Sciences, Chinese Academy of Sciences, 300 Fenglin Road, Shanghai 200032, China; ${ }^{2}$ Zhejiang Academy of Agricultural Sciences, Hangzhou 310021, China

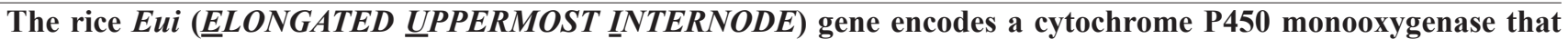
deactivates bioactive gibberellins (GAs). In this study, we investigated controlled expression of the Eui gene and its role in plant development. We found that Eui was differentially induced by exogenous GAs and that the Eui promoter had the highest activity in the vascular bundles. The eui mutant was defective in starch granule development in root caps and Eui overexpression enhanced starch granule generation and gravity responses, revealing a role for GA in root starch granule development and gravity responses. Experiments using embryoless half-seeds revealed that $R A m y 1 A$ and GAmyb were highly upregulated in eui aleurone cells in the absence of exogenous GA. In addition, the GA biosynthesis genes GA3ox1 and GA200x2 were downregulated and GA2ox1 was upregulated in eui seedlings. These results indicate that EUI is involved in GA homeostasis, not only in the internodes at the heading stage, but also in the seedling stage, roots and seeds. Disturbing GA homeostasis affected the expression of the GA signaling genes GID1 (GIBBERELLIN INSENSITIVE DWARF 1), GID2 and SLR1. Transgenic RNA interference of the Eui gene effectively increased plant height and improved heading performance. By contrast, the ectopic expression of Eui under the promoters of the rice GA biosynthesis genes GA3ox2 and GA20ox2 significantly reduced plant height. These results demonstrate that a slight increase in Eui expression could dramatically change rice morphology, indicating the practical application of the Eui gene in rice molecular breeding for a high yield potential.

Keywords: ectopic expression, Eui, gibberellin homeostasis, gravity responses, plant height, rice

Cell Research (2008) 18:412-421. doi: 10.1038/cr.2008.28; published online 12 February 2008

\section{Introduction}

The plant hormone gibberellin (GA) controls diverse biological processes such as stem elongation, seed germination and flowering. Many GA-related mutants have been isolated from the model plant species Arabidopsis thaliana and rice (Oryza sativa L.), and the majority of the underlying genes have been found to encode enzymes

Correspondence: Zuhua He

Tel: +86-21-5492-4121; Fax: +86-21-5492-4015

E-mail: zhhe@sibs.ac.cn

Abbreviations: double-stranded RNA (dsRNA); ELONGATED UPPERMOST INTERNODE (Eui); gibberellin (GA); GIBBERELLIN INSENSITIVE DWARF (GID); overexpression (OX); RNA interference (RNAi); slender rice (SLR); wild type (WT)

Received 27 June 2007; revised 10 September 2007; accepted 20 September 2007; published online 12 February 2008 that are related to GA metabolism or components of GA signaling [1-7]. Many GA mutants show dramatically reduced plant height and hence increased yield potential and lodging resistance of modern cereal varieties, thus greatly contributing to the success of the 'green revolution' [8-10]. Recently, GID1 (GIBBERELLIN INSENSITIVE DWARF 1) was identified as a soluble GA receptor in rice and Arabidopsis [11-13].

In addition to components in GA signaling, endogenous GA levels regulated by the GA metabolism also have an important role in the control of plant development. For example, a mutation in the GA20ox gene ( $s d-1)$ resulted in decreased GA levels in rice, which has been attributed to the rice 'green revolution' gene $[9,10]$. Moreover, ectopic expression of OsGA2oxl under the control of the promoter of the GA biosynthesis gene GA3ox2 resulted in a semi-dwarf phenotype, suggesting the potential for high yield production in rice [14]. Many GA-related genes are feedback or 
feedforward regulated by bioactive GAs, where GA20ox and GA3ox function in GA biosynthesis with feedback regulation, and GA2ox functions in GA catabolism with feedforward regulation by bioactive GAs; GA metabolism is also closely related to GA signaling $[4,15]$.

Bioactive $\mathrm{GA}_{1}, \mathrm{GA}_{4}$, their immediate precursors $\mathrm{GA}_{20}$ and $\mathrm{GA}_{9}$, and $\mathrm{C} 20 \mathrm{GAs}$ are deactivated by GA2-oxidases $[4,15-18]$. However, 2-oxidation is not the only mechanism by which bioactive GAs are deactivated. Our previous study has demonstrated that the rice Eui gene (ELONGATED UPPERMOST INTERNODE), also known as Euil [19], encodes a novel P450 monooxygenase that catalyzes 16 $\alpha, 17$-epoxidation of non-13-hydroxylated GAs to generate bio-inactive $16 \alpha, 17-[\mathrm{OH}]_{2}-\mathrm{GAs}$, representing a novel GA deactivation mechanism in rice [20]. The 16 $\alpha, 17-$ $[\mathrm{OH}]_{2}-\mathrm{GAs}$ were also found in extracts of other plant species, suggesting that $16 \alpha, 17$-epoxidation might participate in GA deactivation by EUI-related enzymes in a variety of plants [20]. The finding that Eui is a GA catabolism gene provides additional evidence that the GA metabolism pathway is a useful target for increasing the agronomic value of crops. Indeed, the eui mutants have been used to genetically improve the heading performance of rice male sterile cultivars in hybrid rice production [21-25].

The elevated expression of the Eui gene dramatically reduces the GA levels, leading to severe dwarf phenotypes in most transgenic plants that constitutively express Eui [20]. Therefore, fine-tuning EUI levels by either ectopic expression or RNA interference (RNAi) could effectively decrease or increase internode elongation, resulting in dwarfed or elongated transgenic plants for different breeding purposes. Interestingly, eui plants are still sensitive to exogenous $\mathrm{GA}_{3}$ application, despite the highly accumulated levels of endogenous bioactive GAs in the mutants [20, 22]. Further experiments are needed to define the details of the mechanisms by which Eui regulates GA homeostasis and thereby GA signaling and other GA-related biological processes.

In this study, we investigated the regulation of Eui gene expression and its role in rice development. We generated transgenic plants that ectopically expressed Eui under the GA20ox2 (GA20ox-Eui) and GA3ox2 (GA3ox-Eui) promoters, and decreased Eui expression through RNAi. We show that the Eui gene was induced by bioactive GAs, and that it was preferentially expressed in the vascular bundles of the elongating internodes. The GA signaling genes GID2, GID1 and SLR1 were repressed in the eui mutant and were upregulated in the Eui-OX transgenic plants. The expression of RAmylA and GAmyb was highly elevated in the eui mutants compared with the wild type (WT), leading to higher $\alpha$-amylase activity in the aleurone layer. Accordingly, starch granule development was defective in the root-tip cells of the eui mutants, whereas it was enhanced in Eui-OX plants, resulting in altered gravity responses. Ectopic expression of the Eui gene driven by the GA gene promoters dramatically decreased plant height in a dosedependent manner. We also discuss how the Eui gene is involved in GA homeostasis and its potential utilization in crop improvement.

\section{Results}

Differential response of eui mutants and WT plants to GAs

eui mutants accumulate extremely high levels of $\mathrm{GA}_{1}$ and $\mathrm{GA}_{4}[20]$. However, our previous studies have shown that eui mutants remain highly sensitive to exogenous $\mathrm{GA}_{3}$ application at the heading stage [22]. To study the responsiveness of eui mutants to exogenous GAs at the seedling stage, we incubated eui-1 and WT plants with bioactive $\mathrm{GA}_{3}, \mathrm{GA}_{1}, \mathrm{GA}_{4}$ and the precursor $\mathrm{GA}_{9}$. As shown in Figure $1 \mathrm{~A}$ and $1 \mathrm{~B}, \mathrm{GA}_{3}$ was most effective in increasing plant height in both WT and eui plants. The eui mutants were significantly more responsive than the WT plants to $\mathrm{GA}_{4}$ and $\mathrm{GA}_{9}$. Because $\mathrm{GA}_{4}$ and $\mathrm{GA}_{9}$ are the substrates of EUI [20], they would not be deactivated by a functional EUI and therefore exhibited greater activity in eui plants. Although both eui and WT plants responded to $\mathrm{GA}_{1}$, no significant difference was observed in their responses to this GA molecule since the control eui plants were found to be slightly taller than WT plants (Figure 1A and 1B). Similar results were also observed with spray treatment of GAs (data not shown). We propose that rice may have a high saturated GA response threshold. In support of this idea, we found a saturated response to about $9 \mathrm{mM} \mathrm{GA}_{3}$ in WT plants at the seedling stage (data not shown).

Since EUI uses $\mathrm{GA}_{4}$ but not $\mathrm{GA}_{1}$ as a substrate, and $\mathrm{GA}_{3}$ is not an endogenous GA, we next tested whether these GAs differentially induce the Eui gene in seedling leaves. We found that Eui expression was induced by $\mathrm{GA}_{4}$ within 1-4 h (Figure 1C). Eui was also induced by $\mathrm{GA}_{1}$ and $\mathrm{GA}_{3}$ in a biphasic pattern that is different from that of $\mathrm{GA}_{4}$ (Figure 1C). This kind of transient GA induction of Eui is probably in agreement with its expression pattern of strong tissue and developmental specificity [20]. Our current study indicates that the Eui gene is also involved in GA homeostasis at the seedling stage (also see below), even though Eui is not expressed at high levels in young seedlings and there is no strong phenotype of the eui mutants at this stage [20].

\section{Eui is highly expressed in vascular cells}

$E u i$ is primarily expressed in rapidly elongating or dividing tissues, including the divisional zone and the 


\section{A}

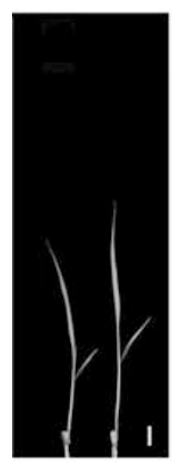

WT eui-1 Mock

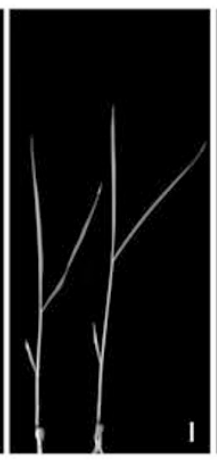

WT eui-1 $\mathrm{GA}_{1}$

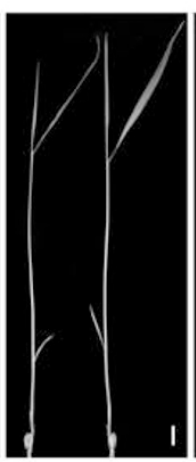

WT eui-1 $\mathrm{GA}_{3}$

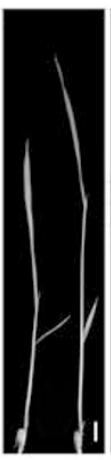

WT eui-1 WT eui-1 $\mathrm{GA}_{4} \quad \mathrm{GA}_{9}$
B

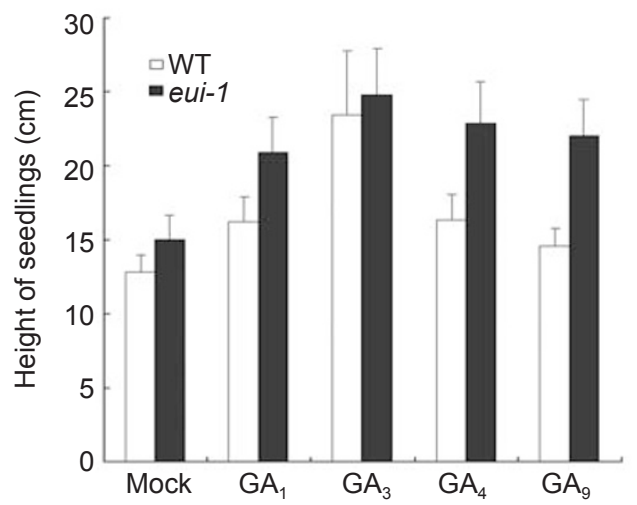

C

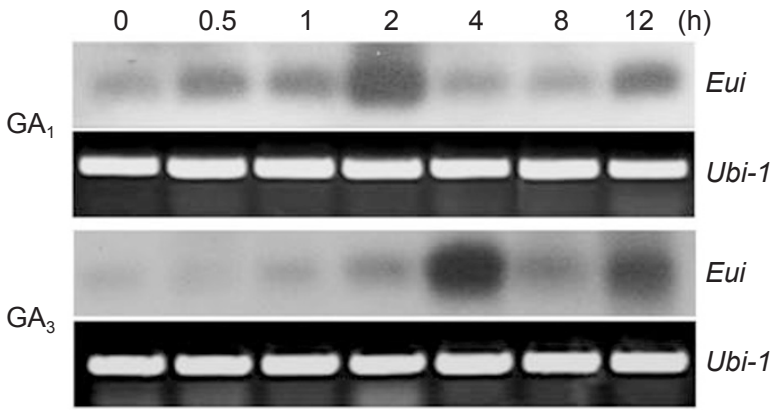

$\mathrm{GA}_{4}$

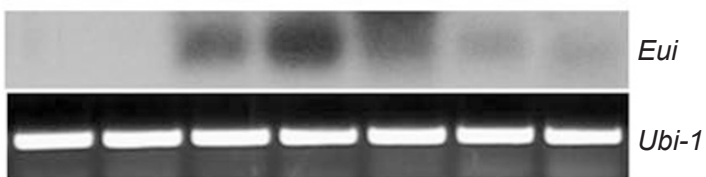

Figure 1 GA sensitivity of eui and wild-type plants and GA induction of Eui. (A) Sensitivity of eui mutant and wild-type (WT) seedlings to different GA molecules. Two-week-old seedlings were incubated with GAs $(1 \mu \mathrm{M})$ or without GAs (mock) for 1 week. (B) Plant elongation of eui and wild-type seedlings treated with GAs. Plant height was measured at day 7 after GA incubation. Error bars show standard errors (SE). (C) GA induction of Eui. Wild-type seedlings were incubated with $\mathrm{GA}_{1}, \mathrm{GA}_{3}$ and $\mathrm{GA}_{4}(1 \mu \mathrm{M})$ for $0-12 \mathrm{~h}$. The RT-PCR products were subjected to DNA gel blot analysis to detect the Eui expression levels. Ubi-1 was used as a control. Results are representative of two independent experiments with similar results. node of the uppermost internode [20]. Bioactive GAs are thought to be produced in these regions because GA20ox2 and GA3ox2 are also predominantly expressed here [26]. To test whether Eui is expressed in specific cells in this region, we analyzed $\beta$-glucuronidase (GUS) staining of cross-sections of the divisional zone of an elongating uppermost internode of the Eui-GUS transgenic plant [20]. Strong GUS staining was observed in the vascular bundles of the elongating internodes (Figure 2A). Interestingly, the highest level of GUS was found in the parenchyma cells (Figure 2B), suggesting that GAs might be produced in or transported through the vascular system.

\section{Eui regulates root starch granule development and gravity responses}

An early study showed that GA plus kinetin treatment could remove starch granules in amyloplasts and therefore change gravisensitivity of cress roots [27]. We were interested in determining whether those Eui-mediated GA phenotypes affect starch accumulation and graviresponsiveness in rice roots. As shown in Figure 3A and $3 \mathrm{~B}$, starch granules were almost completely absent in eui root-tip cells, while their generation was enhanced in the roots of Eui-OX plants compared with WT plants. As a consequence of the altered starch granule development, we observed that Eui-OX roots were more hypersensitive than the WT to gravity. After $2 \mathrm{~h}$ of rotation away from vertical, most Eui-OX root tips bended near vertical (Figure 3C). All WT and Eui-OX root tips bended vertically when roots were rotated over $12 \mathrm{~h}$. These results indicate that Eui is also involved in GA homeostasis in rice roots and reveal a novel role for GA in gravity responses.

\section{GA signaling is regulated by Eui expression}

EUI P450 deactivates bioactive GAs and knockout mutants accumulate high levels of bioactive GAs [20]. Therefore, we next investigated whether the GA signaling
A

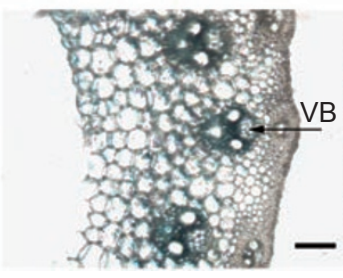

B

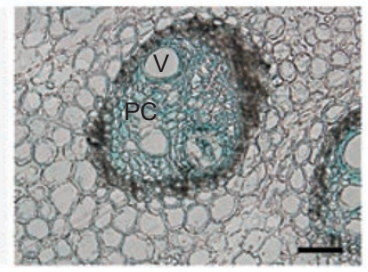

Figure 2 Cell-specific activity of the Eui promoter. (A) GUS staining was detected in the cross-section of the divisional zone of the elongating uppermost internode of the Eui-GUS transgenic plant. Bar $=50 \mu \mathrm{m}$. (B) The highest activity was found in the parenchyma cells (PC) of the vascular bundles (VB). Bar $=200 \mu \mathrm{m}$. V, vessel. 
A

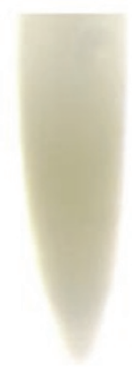

eui-1

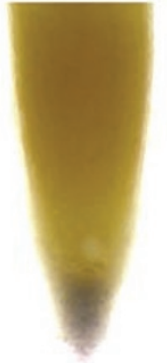

WT

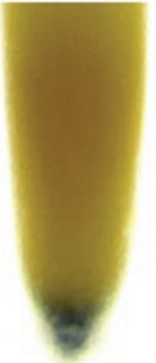

TP309

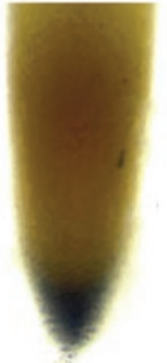

Eui-OX

B

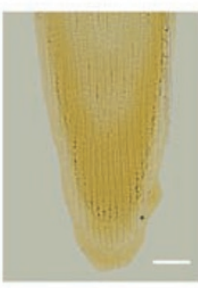

eui-1

C

eui-
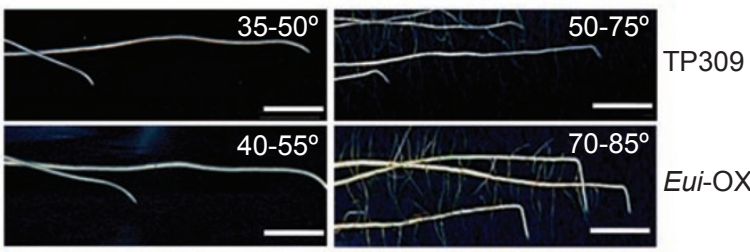

Figure 3 Effects of Eui on root starch granule development and gravity responses. (A) Starch granules in roots of eui mutant, wildtype and Eui-OX and transgenic parent (TP309) plants. Starch granules are stained as black spots in the root tips. Results are representative of three independent experiments with similar results. (B) Longitudinal section of stained seedling root tips. Starch granules are displayed as brown spots. Bar=100 $\mu \mathrm{m}$. (C) Gravisensitivity of seedling roots. Results are representative of two independent experiments with similar results. Bending angles of the majority of roots are indicated for each line. Bar=5 mm.

genes are affected in eui and Eui-OX plants. As shown in Figure 4A, the expression of the positive GA signaling genes, the receptor gene GID1 [11] and the F-box gene GID2 [5] was repressed in the eui mutant and increased in Eui-OX plants. Intriguingly, the negative GA regulator SLR1, which encodes a DELLA protein [3], was slightly repressed in the young panicle of eui plants compared with WT plants. Likewise, overexpression (OX) of Eui slightly increased SLR1 transcription compared with the WT. In support of our observation, SLR1 protein accumulation or stability was greater in transgenic plants constitutively expressing Eui [19]. By contrast, D1, a positive regulator of GA signaling that encodes the $\alpha$-subunit of the heterotrimeric $\mathrm{G}$ protein $[1,2]$, was not affected (Figure 4A). In addition, we observed that the GA biosynthesis genes GA3ox1 and GA20ox2 were downregulated and GA2ox1 was upregulated in the eui seedlings (Figure 4B), similar to their expression in the internode during the heading stage [20]. This result supports the above proposal that Eui also regulates GA homeostasis in the seedling stage.

Aleurone cells recognize GA signals and trigger expression of $\alpha$-amylase [28]. We therefore assayed amylase activity to further characterize the altered GA signaling in eui plants. WT and eui mutant embryoless half-seed plants were placed on starch plates with or without 1 $\mu \mathrm{M} \mathrm{GA}{ }_{3}$ for 3 days, and the starch was then stained with iodine. Production of $\alpha$-amylase from WT half-seeds was observed only on the plate containing $\mathrm{GA}_{3}$. By contrast, eui half-seeds produced strong amylase activity even in the absence of exogenous $\mathrm{GA}_{3}$ (Figure 4C). Similar production of $\alpha$-amylase was observed in the rice slender (slr) mutant [3]. Consistently, we detected higher expression levels of the $\alpha$-amylase gene RAmylA and GAmyb, a positive regulator of $R A m y 1 A$ expression [29], compared with their expression in the WT (Figure 4D). These results demonstrate that amylase activity was high in the eui seeds, which indicates that GA signaling is enhanced in the eui aleurone cells. However, we did not observe decreased $S L R 1$ expression either in the eui aleurone cells or in the eui seedlings (Figure 4B and 4D), which suggests that SLR1 might detect the GA signal differentially in different rice tissues. Together, these results indicate that the GA signaling pathway is affected in eui and Eui-OX plants owing to altered GA homeostasis.

\section{RNAi of Eui effectively increases internode elongation}

The eui phenotype that has increased panicle exertion (heading performance) has been used in breeding for male sterile varieties of hybrid rice [21-25]. From this we developed rice that has an eui phenotype using RNAi. Using a double-stranded RNA (dsRNA) transgenic approach [30], we efficiently generated Eui knockout/knockdown plants, of which more than $80 \%$ of independent transgenic plants showed an elongated internode phenotype with decreased or undetectable expression of Eui that is similar to the eui mutant (in Figure 5A-5C, the results from four representative $\mathrm{T} 1$ transgenic plants are shown). These RNAi lines were stably inherited within generations (data not shown). Consequently, this study provides a feasible approach to rapidly develop elite eui rice lines, which requires a long breeding term when using conventional breeding practices. 
A

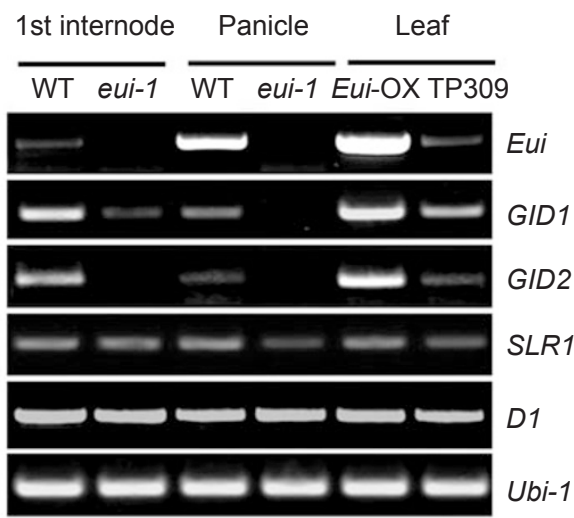

C

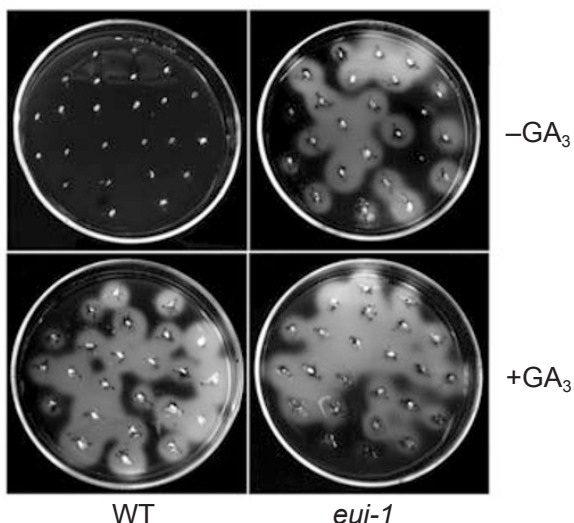

B

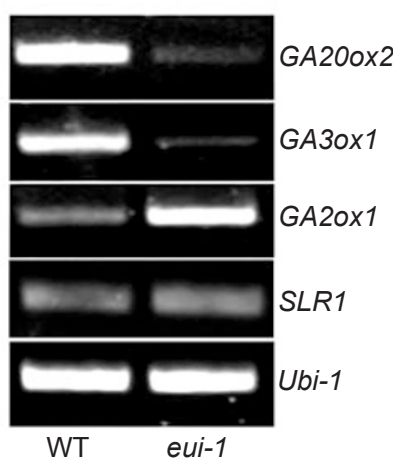

D

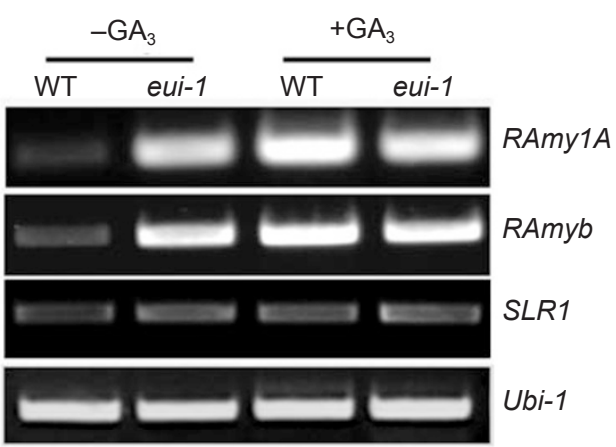

Figure 4 Effect of Eui expression on rice GA signaling. (A) Differential expression of GID1, GID2, SLR1 and D1 in eui mutant, wild-type and Eui-OX plants. Ubi-1 was used as a control during RT-PCR. Results are representative of two independent experiments with similar results. TP309 is the wild type for the Eui-OX transgene. (B) Transcript levels of the rice GA3ox1, GA20ox2, GA2ox 1 and SLR1 in seedlings, as estimated by RT-PCR. Ubi-1 was used as a control. Results are representative of two independent experiments with similar results. (C) Amylase activity in the aleurone cells of the embryoless half-seeds of the wild type and eui mutant. Production of $\alpha$-amylase was detected by staining starch with iodine in plates with or without $1 \mu \mathrm{M} \mathrm{GA}$. Results are representative of two independent experiments with similar results. (D) Transcript levels of the rice RAmy1A, GAmyb and SLR1 in the eui mutant and wild-type aleurone cells with or without treatment with $1 \mu \mathrm{M} \mathrm{GA}_{3}$, as detected by RT-PCR. Ubi-1 was used as a control. Results are representative of two independent experiments with similar results.

Ectopic expression of Eui strongly reduces plant height

The Eui gene dramatically reduces plant height when overexpressed in transgenic rice, leading to severe dwarfing and infertile transgenic plants [20]. We also observed rare Eui-OX transgenic plants that displayed different reductions in height and produced seeds but were unstable within generations (Supplementary information, Figure S1). We confirmed that the height reduction of these lines correlates well with expression levels of Eui (Supplementary information, Figure S1).

In order to exploit the Eui application in rice molecular breeding, we further transformed an elite but tall variety (R2212) with Eui under the control of the GA biosynthesis genes GA20ox2 and GA3ox2, which function in sequential synthesis of GA9/GA20 and $\mathrm{GA}_{1} / \mathrm{GA}_{4}$, respectively [4]. As shown in Figure 6A (where results from four representative transgenic lines are shown), the independent transgenic lines expressing GA20ox-Eui exhibited a range of dwarf phenotypes with normal (lines 1 and 2) or less seed setting (lines 3 and 4), and later flowering. Their phenotypes were also dose-dependent on Eui expression (Figure 6B). Similarly, the transgenic plants with the GA3ox-Eui chimeric gene also exhibited plant height reduction in a dose-dependent manner. (In Figure 7A and 7C, the results from four representative $\mathrm{T} 1$ transgenic plants are shown.) In contrast to GA20ox-Eui plants, GA3ox-Eui plants showed less reduction in plant height, and some lines, such as line 1, exhibited a semi-dwarf phenotype with normal seed setting (Figure 7A and 7B). The different outcomes of the GA20ox-Eui and GA3ox-Eui transgenes are prob- 
A

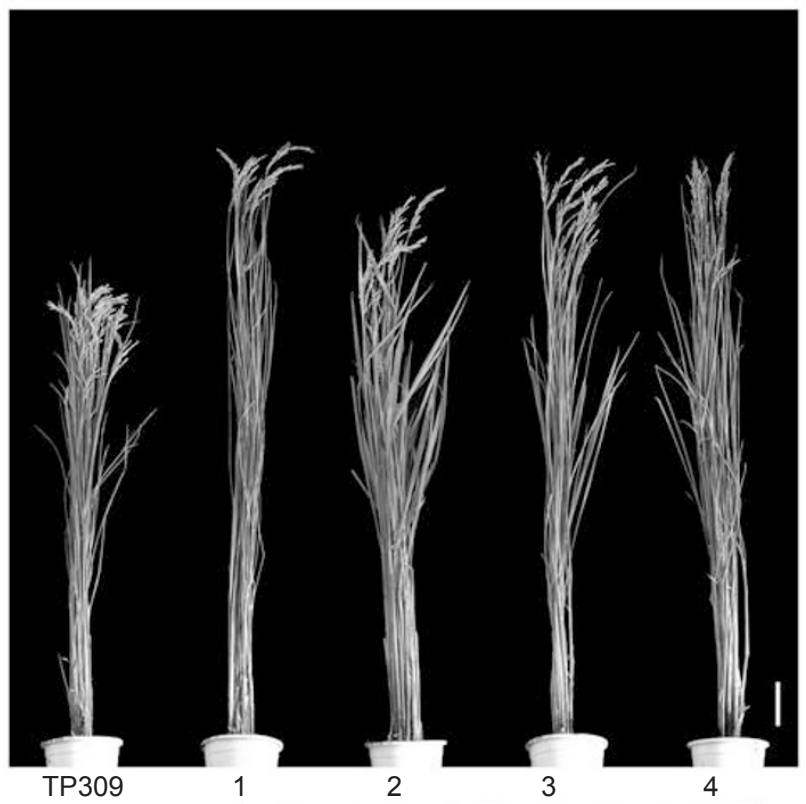

Eui-RNAi

B

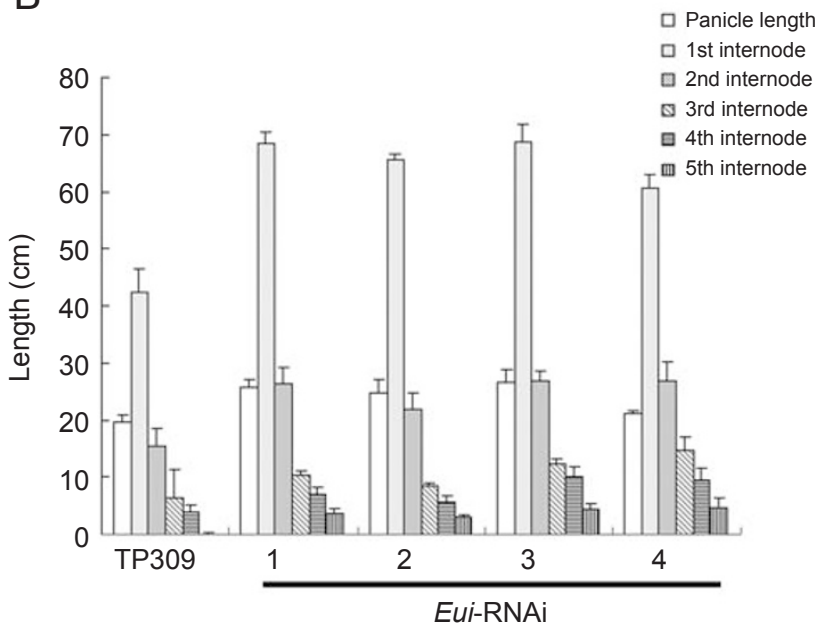

C

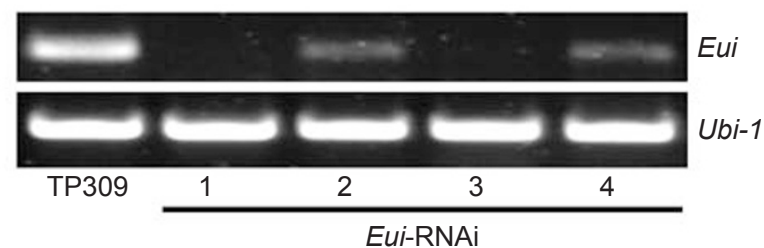

Figure 5 Efficient knockout/knockdown of the Eui gene by RNAi. (A) Morphological phenotypes of RNAi (RNA interference) and wild-type seedlings (inset) and adult plants. Four representative independent RNAi plants (T1) are shown. Bar=10 cm. (B) Lengths of individual internodes of wild-type and RNAi plants. (C) Expression levels of Eui detected by RT-PCR. Ubi-1 was used as a control. ably attributed to the tissue-specific expression patterns of GA3ox2 and GA20ox2 [26]. Similar results were also observed in transgenic plants expressing a GA3ox2-GA2ox 1 chimera [14]. The stable inheritance of these transgenic lines indicates the feasibility of genetic improvement of rice varieties by modulating GA catabolism with the Eui gene. These results also confirm that Eui is a strong regulator of GA homeostasis, which fine-tunes rice plant height in WT plants.

\section{Discussion}

Fine-tuning of GA homeostasis is essential for the establishment of GA-related phenotypes [4, 7, 15]. The amount of bioactive GAs is tightly maintained by both GA synthesis and catabolism. At least in rice, GA catabolism is performed by two types of enzymes: GA2-oxidases that convert bioactive $\mathrm{GA}_{1}, \mathrm{GA}_{4}$, their immediate precursors and

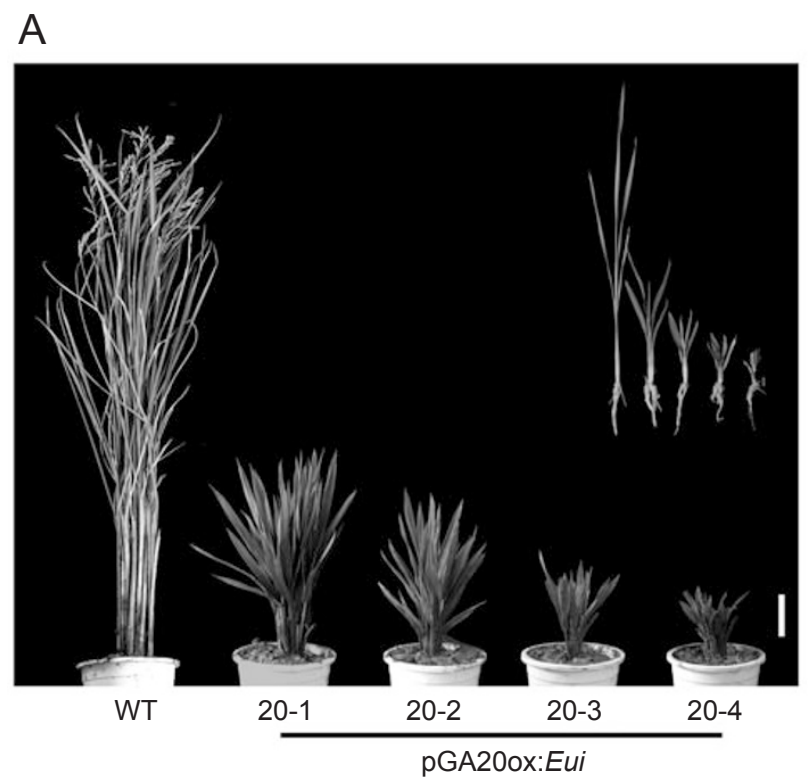

B

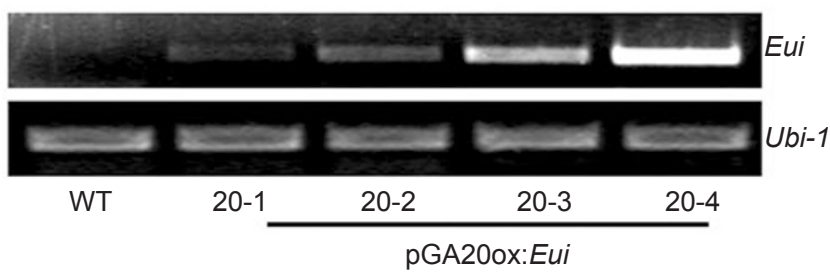

Figure 6 Ectopic expression of Eui under the GA20ox2 promoter in a tall variety, R2212. (A) Morphological phenotypes of wildtype R2212 and GA20ox2-Eui seedlings (inset) and adult plants. Four representative independent transgenic plants are shown. Bar $=10 \mathrm{~cm}$. (B) Expression levels of Eui detected by RT-PCR in GA20ox2-Eui plants. Ubi-1 was used as a control. 
A

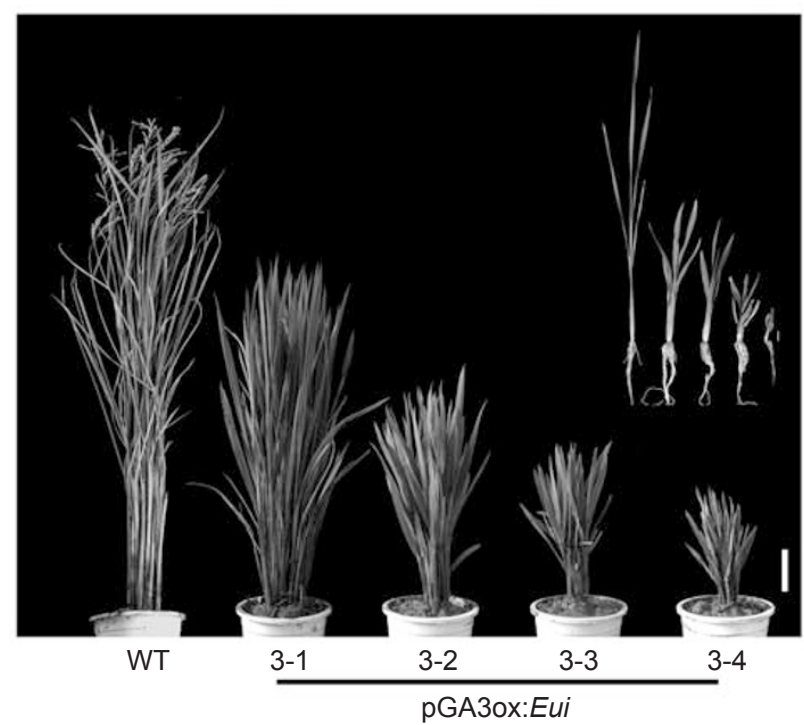

B

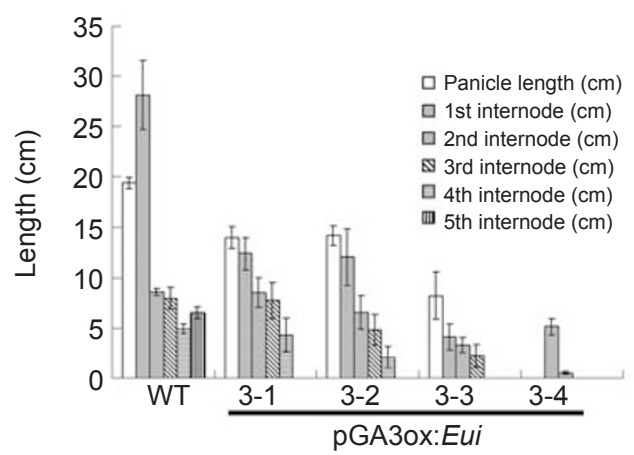

C

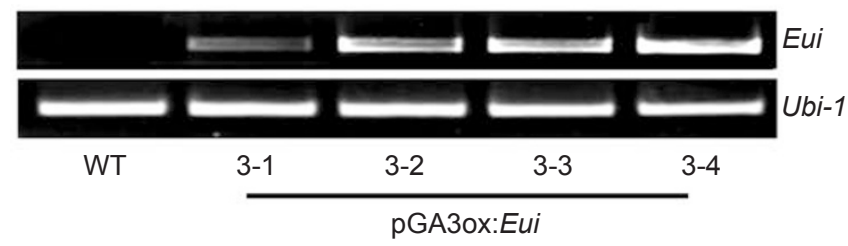

Figure 7 Ectopic expression of Eui under the GA3ox2 promoter in a tall variety, R2212. (A) Morphological phenotypes of wild-type R2212 and GA3ox2-Eui seedlings (insert) and adult plants. Four representative independent transgenic plants (T1) are shown. Bar=10 cm. (B) Lengths of panicles and internodes of wild-type and transgenic plants. (C) Expression levels of Eui detected by RT-PCR in GA3ox2-Eui plants. Ubi-1 was used as a control.

C20 GAs into inactive GAs by $2 \beta$-hydroxylation; and EUI P450 that converts bioactive $\mathrm{GA}_{4}$ and its precursor $\mathrm{GA}_{9}$ into inactive $16 \alpha, 17$ epoxy-GAs by $16 \alpha, 17$-epoxidation. The Eui expression pattern and the eui phenotypes suggest that EUI is a major GA catabolism enzyme in internodes of rice at the heading stage [20]. We have shown that the eui mutant is more sensitive than the WT to $\mathrm{GA}_{4}$ and $\mathrm{GA}_{9}$ at the seedling stage, and both are EUI substrates (Figure $1 \mathrm{~A}$ and $1 \mathrm{~B})$. We have further indicated that the expression of the Eui gene responds to $\mathrm{GA}_{1}, \mathrm{GA}_{3}$ and $\mathrm{GA}_{4}$, although $\mathrm{GA}_{1}$ is not an EUI substrate, and $\mathrm{GA}_{3}$ is not an endogenous $\mathrm{GA}$. We propose that because $\mathrm{GA}_{1}$ and $\mathrm{GA}_{3}$ are structurally similar to $\mathrm{GA}_{4}$, they would exhibit a certain capacity to trigger the Eui promoter with a different pattern (Figure 1C), or, alternatively, that $\mathrm{GA}_{1}$ and $\mathrm{GA}_{3}$ regulate $\mathrm{GA}$ signaling and hence Eui expression (see below). In addition, starch granules were almost devoid in the root tip of the eui mutant and were increased in those of Eui-OX plants, consequently affecting root gravity tropism (Figure 3). Although treatment with GA plus kinetin removed starch granules and thereby altered the gravisensitivity of cress roots [27], a reduction in the amount of GAs in maize seedlings does not significantly alter root graviresponsiveness [31]. Our current study indicates that GA has a role in starch granule generation and gravity tropism in rice roots and suggests that plants might be different in their requirements for GA in graviresponsiveness. It will be interesting to investigate whether mutants related to GA signaling such as gid1, gid2, $\operatorname{slr} l$ and $d l$ also alter gravity tropism. Although there is no significant eui phenotype and detectable $\mathrm{GA}_{1}$ and $\mathrm{GA}_{4}$ at the seedling stage [20], we have found that the GA metabolism genes are indeed either feedback or feedforward regulated at this stage (Figure 4B). Collectively, our current study demonstrates that Eui is involved in GA homeostasis, not only at the heading stage but also at the seedling stage and in roots.

The Eui gene is also inducible by $\mathrm{GA}_{3}$, a non-endogenous GA, although with a different pattern to induction by $\mathrm{GA}_{4}$. Similar $\mathrm{GA}_{3}$ induction was also observed for the $\mathrm{Ara}$ bidopsis GA2ox genes [16]. However, the rice OsGA2oxl gene does not respond to exogenous $\mathrm{GA}_{3}$ [32], suggesting that rice GA catabolism genes might differentially respond to this GA molecule. Detailed analysis of the structures and cis-elements of their promoters would provide further information about their regulation in GA responses. Interestingly, strong Eui-GUS expression was observed in vascular bundles (Figure 2). A similar cell-specific pattern was also observed for OsGA2oxl [32]. It is well established that GAs are produced in certain tissues, particularly in young panicles in rice [33]. However, there is no information about how bioactive GAs are delivered to other tissues/sites. Our current study raises the possibility that GAs might be transported through the vascular system.

Co-expression of GA biosynthesis and signaling genes in rice tissues indicates that the biosynthesis of active GAs occurs at the same site as GA signaling [26]. The Eui expression pattern also suggests that the sites of GA deactivation by EUI and the synthesis of bioactive GAs may partially 
overlap at the heading stage [20]. We have shown in this study that Eui is involved in GA homeostasis, and therefore modulates the expression of the genes that are involved in GA signaling and $\alpha$-amylase production (Figures 3 and 4). Embryoless half-seeds are used to eliminate the effect of the embryo (the site of GA production), so they are usually GA-deficient. Interestingly, the embryoless halfseeds of the eui mutant continued to produce and secrete large amounts of $\alpha$-amylase (Figure $4 \mathrm{C}$ ). Because no GA biosynthesis is observed in the rice aleurone layer [26], it is possible that the elevated GA levels in maturing eui seeds induce amylase expression and accumulation before the seed fully desiccates. An alternative explanation is that a trace amount of bioactive GAs might be present in the aleurone layer during seed development in the eui mutant so that once the seeds are imbibed they produce $\alpha$-amylase without GA treatment. In support of the first possibility, the GA2ox-deficient mutant M326 of barley accumulates high levels of $\mathrm{GA}_{1}$, resulting in high levels of premature $\alpha$-amylase expression [34].

Surprisingly, we found that the expression of both positive GA signaling genes GID1 and GID2 and the negative signaling gene SLR1 was repressed in the eui mutant internodes and increased in plants overexpressing Eui (Figure 4A). We propose that EUI influences the GA signaling network due to altered GA levels according to a feedback mechanism that is similar to that operating in GA biosynthesis. It is possible that the eui mutant plants accumulate high levels of bioactive GAs, and that the overproduction of bioactive GAs could feedback regulate GA signaling by decreasing the production of GID1 and GID2, since GA treatment reduced transcript abundance for all three GID1 genes of Arabidopsis [13]. Such high GA levels could also repress SLR1 expression as SLR1 protein accumulation was decreased or eliminated by GA treatment [5]. However, the proposed GA-mediated SLRI transcription and relationship between these regulation events need more study [13], as SLR1 expression was not altered in the eui seedlings and aleurone cells compared with the WT in our current study.

Breeding cereal crops for a desirable plant height or morphology has been a goal of the agriculture industry. In rice, male sterile cultivars are commonly defective in elongation of the uppermost internode owing to inadequate GA production in the empty anthers [35]. The eui mutation provides a tool for genetically improving the heading performance of male sterile cultivars, and rice varieties carrying the eui mutation have been officially released in China [21-25]. However, traditional breeding of eui varieties takes a long time because of its recessive nature. We efficiently developed eui rice using an RNAi transgene (Figure 5), and we are currently using this approach to develop elite eui male sterile lines. On the other hand, dwarf architecture, or the so-called 'green revolution', has been valuable in rice breeding, which confers lodging resistance and high yield potential. Genetic manipulation of the levels of bioactive GA is a practical strategy to generating rice with suitable plant height for high yield. Two approaches can be adopted to lower endogenous GA levels and therefore develop elite varieties; these include decreasing GA biosynthesis and increasing GA catabolism. Indeed, by using the mutant GA biosynthesis gene $s d-1$, a mutated GA20ox gene $[9,10]$, or by using the GA catabolism gene OsGA2ox 1 to manipulate GA levels [14], breeding for semi-dwarf rice is now feasible through molecular design.

In our previous study, we developed Eui-OX transgenic plants that showed a severe dwarf phenotype with defects in flower development [20], and the semi-dwarf plants generated were genetically unstable (Supplementary information, Figure S1), limiting the use of the constitutive expression approach in rice molecular breeding. We therefore generated transgenic plants of a tall variety with GA20ox-Eui and $G A 3 o x$-Eui fusions (Figures 6 and 7). In contrast to the GA20ox-Eui transgenic approach, the GA3ox-Eui fusion produced more desirable semi-dwarf lines with normal seed setting. The same GA3ox2 (D18) promoter was also used to drive GA2oxl expression and to generate semi-dwarf plants [14]. This approach can also be used in other cereals such as maize and barley. Regardless, this study suggests that manipulation of the Eui gene could improve rice varieties through the modulation of GA catabolism.

\section{Materials and Methods}

\section{$G A$ response assay}

Two-week-old WT (ZS97) and eui-1 mutant plants were incubated in $1 / 2 \mathrm{MS}$ medium containing $1 \mu \mathrm{M} \mathrm{GA}_{1}, \mathrm{GA}_{3}, \mathrm{GA}_{4}$ and $\mathrm{GA}_{9}$; seedling height (from the base to the leaf tip) was measured at day 7 after GA treatment. For GA induction of Eui, seedlings were treated with $1 \mu \mathrm{M} \mathrm{GA}, \mathrm{GA}_{3}$ and $\mathrm{GA}_{4}$, and samples were collected at different time points for RNA isolation.

\section{Amylase activity}

Matured seeds of the WT and the eui-1 mutant were dried and stored for 3 months. Embryoless half-seeds were sterilized in 3\% $\mathrm{NaClO}$ for $15 \mathrm{~min}$, washed with sterile water three times, and then incubated on agar plates containing $0.2 \%$ starch with or without 1 $\mu \mathrm{M} \mathrm{GA}_{3}$ for 3 days at $28^{\circ} \mathrm{C}$ in darkness. The plates were exposed to iodine vapor to determine $\alpha$-amylase activity as described previously [36]. The same treated half-seeds were collected at day 2 for RNA preparation to detect RAmy1A, GAmyb and SLRI expression.

\section{GUS staining}

Histochemical assays for GUS activity in transgenic plants were performed as described previously [37]. Transverse sections $(4 \mu \mathrm{m})$ of the divisional zone of the elongating uppermost internode of the Eui-GUS transgenic plant [20] were observed and photographed 
under a microscope (Olympus, Tokyo, Japan).

\section{Detection of starch granules and root gravitropism}

Seedlings were grown in 1/2 MS medium. Root starch granule staining was performed as described previously [38]. Longitudinal sections $(2 \mu \mathrm{m})$ of stained root tips were examined and photographed under a microscope (Olympus, Tokyo, Japan). For gravitropism analysis, 2-week-old seedlings grown in 1/2 MS agar were rotated by $90^{\circ}$. The root tip positions were recorded at $2 \mathrm{~h}$ after rotation.

\section{Transgenic constructs and rice transformation}

OX of Eui has been described previously [20]. The entire RNAi cassette of sense and antisense fragments of Eui was cloned into the vector pCAMBIA1300 s (provided by Dr Yinong Yang) [30], and was introduced into the WT variety TP309 to generate more than 25 independent plants by Agrobacterium tumefaciens-mediated transformation. Progeny plants (T1 up to T3) were assayed. For the ectopic expression of Eui, the promoter regions that were $2.0 \mathrm{~kb}$ upstream of the coding regions of GA20ox2 ( $S d 1$, accession number AF465255) and $1.38 \mathrm{~kb}$ upstream of the coding regions of GA3ox2 (D18, accession number P0013F10.29) were fused to the full-length Eui cDNA (accession number AY987040) to generate GA20ox-Eui and GA3ox-Eui fusions. These were inserted into the vector pCAMBIA1300 (accession number AF234269). The plasmids were transformed into a tall japonica variety R2212 by Agrobacterium tumefaciens-mediated transformation to generate more than 30 independent transgenic lines for each construct. T0 (severe dwarf, no seed setting) or T1 (seed setting) progeny plants were analyzed.

\section{$R N A$ preparation and transcript analysis}

Total RNA was isolated from treated tissues or transgenic plant stems using TRIzol reagent according to the manufacturer's protocol (Invitrogen, Carlsbad, CA). The Eui transcripts were detected by RT-PCR and Southern blot as described previously [20]. The transcripts of RAmy1, GAmyb, GID1, GID2, SLR1 and D1 were detected by reverse transcriptase PCR (RT-PCR) with the following primers: Ramy-F 5'-CGCGTCGCACCGAAGCAGAGTA-3'; Ramy-R 5'-AGCAGAGCATCCAGCCCACA-3'; GAMB-F 5'-CATGTAATACTACGGTTCTTAGCC-3'; GAMB-R 5'-GAATCTGCTTTAGCGTCTGG-3'; GID1-F 5'-GAGGTCAACCGCAACGAGTGC3'; GID1-R 5'-GCTGCCGCCGTGGAAGAATA-3'; GID2-F 5'-CGGGGAGGACCTGGTGTTCG-3'; GID2-R 5'-CCCCTCCATTCTTATCACTGTCATTT-3'; SLR1-F 5'-GGTGCGGCCAAGGATCGTCA-3'; SLR1-R 5'-AGGAGCGTGCTCGCCTGTTT-3'; D1-F 5'-AAGGAGGATGTGCTTTATG-3'; and D1-R 5'-TGGTCTAGGGCCGTAGTT-3', using the following PCR conditions: $94{ }^{\circ} \mathrm{C}$ for $4 \mathrm{~min}$, followed by 28 cycles of $94{ }^{\circ} \mathrm{C}$ for $30 \mathrm{~s}, 60^{\circ} \mathrm{C}$ for $30 \mathrm{~s}$, and $72{ }^{\circ} \mathrm{C}$ for $30 \mathrm{~s}$, and an elongation step at $72{ }^{\circ} \mathrm{C}$ for $10 \mathrm{~min}$. The transcripts of GA20ox, GA3Ox and GA2Ox were detected as described previously [20].

\section{Acknowledgments}

We are grateful to Dr Shinjiro Yamaguchi (RIKEN, Japan) for critical reading of the manuscript, and to Professor Yinong Yang (Penn. State University, USA) for the rice RNAi vector. This work was supported by grants from the National Natural Science Foundation of China (30670186 and 30421001), and the Ministry of Science and Technology of China (2006AA10A102) to ZH.

\section{References}

1 Ashikari M, Wu J, Yano M, Sasaki T, Yoshimura A. Rice gibberellin-insensitive dwarf mutant gene Dwarf 1 encodes the alpha-subunit of GTP-binding protein. Proc Natl Acad Sci USA 1999; 96:10284-10289.

2 Ueguchi-Tanaka M, Fujisawa Y, Kobayashi M, et al. Rice dwarf mutant $d 1$, which is defective in the alpha subunit of the heterotrimeric $\mathrm{G}$ protein, affects gibberellin signal transduction. Proc Natl Acad Sci USA 2000; 97:11638-11643.

3 Ikeda A, Ueguchi-Tanaka M, Sonoda Y, et al. slender rice, a constitutive gibberellin response mutant, is caused by a null mutation of the SLR1 gene, an ortholog of the height-regulating gene GAI/RGA/RHT/D8. Plant Cell 2001; 13:999-1010.

4 Olszewski N, Sun TP, Gubler F. Gibberellin signaling: biosynthesis, catabolism, and response pathways. Plant Cell 2002; 14: S61-S80.

5 Sasaki A, Itoh H, Gomi K, et al. Accumulation of phosphorylated repressor for gibberellin signaling in an F-box mutant. Science 2003; 299:1896-1898.

6 Sakamoto T, Miura K, Itoh H, et al. An overview of gibberellin metabolism enzyme genes and their related mutants in rice. Plant Physiol 2004; 134:1642-1653.

7 Sun TP, Gubler F. Molecular mechanism of gibberellin signaling in plants. Annu Rev Plant Biol 2004; 55:197-223.

8 Peng J, Richards DE, Hartley NM, et al. 'Green revolution' genes encode mutant gibberellin response modulators. Nature 1999; 400:256-261.

9 Sasaki A, Ashikari M, Ueguchi-Tanaka M, et al. Green revolution: a mutant gibberellin-synthesis gene in rice. Nature 2002; 416:701-702.

10 Spielmeyer W, Ellis MH, Chandler PM. Semidwarf(sd-1), "green revolution" rice, contains a defective gibberellin 20-oxidase gene. Proc Natl Acad Sci USA 2002; 99:9043-9048.

11 Ueguchi-Tanaka M, Ashikari M, Nakajima M, et al. GIBBERELLIN INSENSITIVE DWARF1 encodes a soluble receptor for gibberellin. Nature 2005; 437:693-698.

12 Nakajima M, Shimada A, Takashi Y, et al. Identification and characterization of Arabidopsis gibberellin receptors. Plant $J$ 2006; 46:880-889.

13 Griffiths J, Murase K, Rieu I, et al. Genetic characterization and functional analysis of the GID1 gibberellin receptors in Arabidopsis. Plant Cell 2006; 18:3399-3414.

14 Sakamoto T, Morinaka Y, Ishiyama K, et al. Genetic manipulation of gibberellin metabolism in transgenic rice. Nat Biotechnol 2003; 21:909-913.

15 Hedden P, Phillips AL. Gibberellin metabolism: new insights revealed by the genes. Trends Plant Sci 2000; 5:523-530.

16 Thomas SG, Phillips AL, Hedden P. Molecular cloning and functional expression of gibberellin 2-oxidases, multifunctional enzymes involved in gibberellin deactivation. Proc Natl Acad Sci USA 1999; 96:4698-4703.

17 Schomburg FM, Bizzell CM, Lee DJ, Zeevaart JAD, Amasino RM. Overexpression of a novel class of gibberellin 2-oxidases decreases gibberellin levels and creates dwarf plants. Plant Cell 2003; 15:151-163. 
18 Lee DJ, Zeevaart JAD. Molecular cloning of GA 2-oxidase 3 from spinach and its ectopic expression in Nicotiana sylvestris. Plant Physiol 2005; 138:243-254.

19 Luo A, Qian Q, Yin H, et al. Euil, encoding a putative cytochrome P450 monooxygenase, regulates internode elongation by modulating gibberellin responses in rice. Plant Cell Physiol 2006; 47:181-191.

20 Zhu Y, Nomura T, Xu Y, et al. ELONGATED UPPERMOST INTERNODE encodes a cytochrome P450 monooxygenase that epoxidizes gibberellins in a novel deactivation reaction in rice. Plant Cell 2006; 18:442-456.

$21 \mathrm{He} Z$, Shen Z. Inheritance of panicle exertion and improvement of male sterile line in rice. Chin J Rice Sci 1991; 5:1-6.

$22 \mathrm{He} Z$, Shen Z. Sensitivity of elongated internode gene to $\mathrm{GA}_{3}$ and improvement of MS line in rice. Acta Agron Sin 1994; 20:161167.

23 He Z, Shen Z. Interaction between eui gene and WAMS cytoplasm of rice and improvement of panicle exsertion of MS line. SABRAO J 1989; 6:753-756.

24 Yang R, Zhang S, Huang R, Yang S, Zhang Q. Breeding technology of eui hybrids of rice. Sci Agric Sin 2002; 35:233-237.

25 Liu L, van Zanten L, Shu Q, Maluszynski M. Officially released mutant varieties in China. Mutat Breed Rev 2004; 14:1-64.

26 Kaneko M, Itoh H, Inukai Y, et al. Where do gibberellin biosynthesis and gibberellin signaling occur in rice plants? Plant $J$ 2003; 35:104-115.

27 Busch MB, Sievers A. Hormone treatment of roots causes not only a reversible loss of starch but also of structural polarity in statocytes. Planta 1990; 181:358-364.

28 Gubler F, Kalla R, Roberts JK, Jacobsen JV. Gibberellin-regulated expression of a $m y b$ gene in barley aleurone cells: evidence for Myb transactivation of a high-pI $\alpha$-amylase gene promoter. Plant Cell 1995; 7:1879-1891.
29 Washio K. Functional dissections between GAMYB and Dof transcription factors suggest a role for protein-protein associations in the gibberellin-mediated expression of the RAmy $1 A$ gene in the rice aleurone. Plant Physiol 2003; 133:850-863.

30 Mei C, Zhou X, Yang Y. Use of RNA interference to dissect defense-signaling pathways in rice. Methods Mol Biol 2006; 354:161-172.

31 Moore R, Dickey K. Growth and graviresponsiveness of primary roots of Zea mays seedlings deficient in abscisic acid and gibberellic acid. J Exp Bot 1985; 36:1793-1798.

32 Sakamoto T, Kobayashi M, Itoh H, et al. Expression of a gibberellin 2-oxidase gene around the shoot apex is related to phase transition in rice. Plant Physiol 2001; 125:1508-1516.

33 Kobayashi M, Yamaguchi I, Murofushi N, Ota Y, Takahashi N. Fluctuation and localization of endogenous gibberellins in rice. Agric Biol Chem 1988; 52:1189-1194.

34 Green LS, Faergestad EM, Poole A, Chandler PM. Grain development mutants of barley ( $\alpha$-amylase production during grain maturation and its relation to endogenous gibberellic acid content). Plant Physiol 1997; 114:203-212.

35 Li J, Yuan L. Hybrid Rice: Genetics, breeding and seed production. Plant Breed Rev 1999; 17:17-158.

36 Yamaguchi J. Analysis of embryo-specific alpha-amylae using isolated mature rice embryos. Breed Sci 1998; 48:365-370.

37 Jefferson RA, Kavanagh TA, Bevan MV. GUS fusions: $\beta$-glucuronidase as a sensitive and versatile gene fusion marker in higher plants. EMBO J 1987; 6:3901-3907.

38 Willemsen V, Wolkenfelt H, de Vrieze G, Weisbeek P, Scheres B. The HOBBIT gene is required for formation of the root meristem in the Arabidopsis embryo. Development 1998; 125:521-531.

(Supplementary Information is linked to the online version of the paper on the Cell Research website.) 